\title{
MAKING SPACE FOR SHAPE
}

Science doesn't quite know what to do with people like Graham Parkhouse, a British engineer who surely qualifies as a maverick. While shoehorned into a brief academic career at the University of Surrey - he now runs a civil engineering consultancy nearby Parkhouse developed ideas on materials selection and design ${ }^{1}$ that proved influential on pioneers of the field such as Michael Ashby and that now seem rather prescient. (Sadly, the same cannot really be said for his published ideas on cosmic background radiation.) Parkhouse promoted the idea of structure as an interplay of material and shape that can now be seen to foreshadow notions of hierarchical materials and metamaterials. His unusual career trajectory is described in Donald Braben's book on blue-skies thinking, Scientific Freedom: The Elixir of Civilization (Wiley, 2008).

Parkhouse's little-remembered contribution is brought to mind in a recent exploration by Barthelat and Mirkhalaf of how material and shape interact ${ }^{2}$. The authors have taken an unusual approach to a well-studied issue: how best to configure a composite to achieve an ideal compromise between the mechanical properties of its constituents. As Barthelat and Mirkhalaf point out, while it is well known that combining a hard and a softer material can engender a balance of stiffness and strength (from the hard component) with toughness and ductility (from the soft), typically in engineering only a few microstructures are employed, such as fibre composites and laminates. Nature is similarly conservative with its own microstructural repertoire, favouring in particular the staggered layering seen in nacre and bone. But are these really the best, or even the only, options?

The design of microstructure is typically conducted as an optimization process that begins with a certain topology and refines it. A more exhaustive search of the space of microstructural possibilities is generally thought computationally prohibitive. But Barthelat and Mirkhalaf describe a model for which this kind of blanket survey is tractable: a two-dimensional composite in which hard, rectangular inclusions are regularly stacked within a softer matrix, subjected to extensional stress. Simple parametrization of this geometry gives rise to just over 7,000 microstructures for a particular choice of the hard (brittle) and soft (elastic) phases. The stress-strain curves and failure of each of these solutions can then be calculated.

Most (about 90 per cent) of the resulting composites are either very brittle - they contain continuous hard phases, which yield by brittle failure - or very ductile, failing by over-extension of the soft matrix. But the remaining structures look more useful. Half of these are 'quasi-brittle': stiff but ductile, failing at strains much greater than that supported by the hard

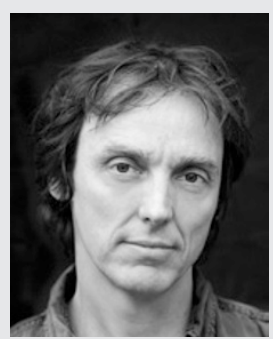

PHILIP BALL

phase alone. The others are labelled 'ductile strong', and include staggered hard platelets comparable to nacre.

The results confirm some expected general principles: stiffness and strength usually come together, whereas strength and toughness tend to be mutually exclusive. With the microstructural space fully mapped, however, it becomes possible to answer design questions rather precisely: to find exactly which shapes achieve a particular balance of properties (if, say, toughness were to be weighted more than stiffness). The approach could be extended to include, for example more degrees of freedom: dissipation by delamination, hierarchical structure and anisotropy. It might even answer questions about natural design: are nature's composites truly optimal, or constrained by their history?

\section{References}

1. Parkhouse, J. G. in Proc. 3rd Int. Conf. Space Structures (ed. Nooshin, H.) 367 (Elsevier Applied Science, 1984)

2. Barthelat, F. \& Mirkhalaf, M. J. R. Soc. Interface 10, 20130711 (2013).

\section{Perovskites under the Sun}

Mixed-halide organic-inorganic hybrid perovskites are reported to display electron-hole diffusion lengths over $1 \mu \mathrm{m}$. This observation provides important insight into the charge-carrier dynamics of this class of semiconductors and increases the expectations for highly efficient and cheap solar cells.

\section{Maria Antonietta Loi and Jan C. Hummelen}

$\mathrm{M}$ aterials that have a generic chemical formula $\mathrm{ABX}_{3}$ and a cubic structure are defined as perovskites, named after the mineral $\mathrm{CaTiO}_{3}$. The $\mathrm{A}$ and $\mathrm{B}$ sites can accommodate inorganic cations of various valency and ionic radius. Alternatively, suitable organic species can replace cation A and create organic-inorganic hybrid materials ${ }^{1}$ (Fig. 1). A number of exciting physical properties, like colossal magnetoresistance, ferroelectricity and superconductivity, have been discovered in this prolific family of compounds during the past century ${ }^{1,2}$. Recently, organic-inorganic hybrid perovskites (in particular $\mathrm{CH}_{3} \mathrm{NH}_{3} \mathrm{PbX}_{3}$, where $\mathrm{X}=\mathrm{I}, \mathrm{Cl}$, $\mathrm{Br}$ ) came to the fore as a result of their 